\title{
Exotic Galilean Symmetry and Non-Commutative Mechanics ${ }^{\star}$
}

\author{
Peter A. HORVÁTHY ${ }^{\dagger}$, Luigi MARTINA $\ddagger$ and Peter C. STICHEL $\S$ \\ $\dagger$ Laboratoire de Mathématiques et de Physique Théorique, Université de Tours, \\ Parc de Grandmont, F-37200 Tours, France \\ E-mail: horvathy@lmpt.univ-tours.fr \\ ¥ Dipartimento di Fisica - Università del Salento and Sezione INFN di Lecce, \\ via Arnesano, CP. 193, I-73100 Lecce, Italy \\ E-mail: Luigi.Martina@le.infn.it \\ $\S$ An der Krebskuhle 21 D-33 619 Bielefeld, Germany \\ E-mail: peter@physik.uni-bielefeld.de
}

Received March 23, 2010, in final form July 19, 2010; Published online July 26, 2010

doi:10.3842/SIGMA.2010.060

\begin{abstract}
Some aspects of the "exotic" particle, associated with the two-parameter central extension of the planar Galilei group are reviewed. A fundamental property is that it has non-commuting position coordinates. Other and generalized non-commutative models are also discussed. Minimal as well as anomalous coupling to an external electromagnetic field is presented. Supersymmetric extension is also considered. Exotic Galilean symmetry is also found in Moyal field theory. Similar equations arise for a semiclassical Bloch electron, used to explain the anomalous/spin/optical Hall effects.
\end{abstract}

Key words: noncommutative spaces; Galilean symmetry; dynamical systems; quantum field theory

2010 Mathematics Subject Classification: 46L55; 37K65; 37L20; 83C65; 81T70

\section{Introduction: "exotic" Galilean symmetry}

A curious property of massive non-relativistic quantum systems is that Galilean boosts only act up-to phase, so that only the 1-parameter central extension of the Galilei group acts unitarily [1]. True representations only arise for massless particles.

Do further extension parameters exist? It is well-known that central extensions are associated with non-trivial Lie algebra cohomology [2,3], and Bargmann [4] has shown that, in $d \geq 3$ space dimensions, the Galilei group only admits a 1-parameter central extension, identified with the physical mass, $m$. Lévy-Leblond [5] has recognized, however, that, owing to the Abelian nature of planar rotations, the planar Galilei group admits a second central extension. The cohomology is two-dimensional, and is parametrized by two constants, namely by the mass and a second, "exotic", parameter $\kappa$. The second extension is highlighted by the non-commutativity of Galilean boost generators,

$$
\left[K_{1}, K_{2}\right]=i \kappa
$$

This fact has long been considered, however, a mere mathematical curiosity, as planar physics itself has been viewed as a toy.

\footnotetext{
*This paper is a contribution to the Special Issue "Noncommutative Spaces and Fields". The full collection is available at http://www.emis.de/journals/SIGMA/noncommutative.html
} 
Around 1995 the situation started to change, though, with the construction of physical models carrying such an "exotic" structure $[6,7,8]$. [8] uses an acceleration-dependent Lagrangian, while that in [9], is obtained following Souriau's method [3].

These models have the distinctive feature that the Poisson bracket of the planar coordinates does not vanish,

$$
\left\{x_{1}, x_{2}\right\}=-\frac{\kappa}{m^{2}} \equiv \theta,
$$

and provide us with two-dimensional examples of non-commutative mechanics $[10,11,12,13$, $14,15]^{1}$.

What is the physical origin of exotic mechanics? What is the quantum mechanical counterpart? An answer has been found soon after its introduction: it is a sort of "non-relativistic shadow" of (fractional) spin $[16,17,18]$. Our particles can be interpreted therefore, as nonrelativistic anyons $[19,20]$.

The supersymmetric extension of the theory is outlined in Section 9.

All these examples have been taken from one-particle mechanics; exotic Galilean symmetry can be found, however, also in field theory [21], as explained in Section 10.

Remarkably, similar structures were considered, independently and around the same time, in condensed matter physics, namely for the Bloch electron [22]: it was argued that the semiclassical dynamics should involve a "Berry term", which induces "anomalous" velocity similar to the one in the "exotic" model [9].

These, 3 -space dimensional, models are also non-commutative, but the parameter $\theta$ is now promoted to a vector-valued function of the quasi-momentum: $\vec{\Theta}=\vec{\Theta}(\vec{k})$. Exotic Galilean symmetry, strictly linked to two space dimensions, is lost. However, a rich Poisson structure and an intricate interplay with external magnetic fields can be studied. Further developments include the Anomalous [23], the Spin [24] and the Optical [25, 26, 27, 28] Hall effects.

This review completes and extends those in [29, 30, 31].

\section{Exotic model, constructed by Souriau's method}

Models associated with a given symmetry group can be conveniently constructed using Souriau's method: the classical phase spaces of "elementary systems" correspond to coadjoint orbits of their symmetry groups [3]. This was precisely the way followed in [6, 9] to produce an "elementary" classical system carrying Lévy-Leblond's "exotic" Galilean symmetry. Translated from Souriau's to more standard terms, the model has an "exotic" symplectic form and a free Hamiltonian,

$$
\begin{aligned}
\Omega_{0} & =d p_{i} \wedge d x_{i}+\frac{1}{2} \theta \epsilon_{i j} d p_{i} \wedge d p_{j}, \\
H_{0} & =\frac{\vec{p}^{2}}{2 m} .
\end{aligned}
$$

The associated free motions follow the usual straight lines, described by the equations

$$
m \dot{x}_{i}=p_{i}-m \theta \epsilon_{i j} \dot{p}_{j}, \quad \dot{p}_{i}=0 .
$$

The "exotic" structure only enters the conserved quantities, namely the boost and the angular momentum,

$$
j=\epsilon_{i j} x_{i} p_{j}+\frac{\theta}{2} \vec{p}^{2}, \quad K_{i}=-m x_{i}+p_{i} t-m \theta \epsilon_{i j} p_{j} .
$$

\footnotetext{
${ }^{1}$ Our conventions are as follows. Symbols with arrows denote vectors, and those in boldface are tensors. The position vector $\vec{r}$ has coordinates $x_{i}$.
} 
The "exotic" structure behaves hence roughly as spin: it contributes to some conserved quantities, but the new terms are separately conserved. The new structure does not seem to lead to any new physics.

The situation changes dramatically, though, if the particle is coupled to a gauge field. Applying Souriau's coupling prescription [3] yields indeed

$$
\Omega=\Omega_{0}+e B d q_{1} \wedge d q_{2}, \quad H=H_{0}+e V,
$$

where $B$ is the magnetic field assumed to be perpendicular to the plane, and $V$ is the electric potential. For simplicity, both $B$ and $V$ are assumed to be time-independent.

The associated Poisson bracket then automatically satisfies the Jacobi identity. The resulting equations of motion read

$$
m^{*} \dot{x}_{i}=p_{i}-e m \theta \epsilon_{i j} E_{j}, \quad \dot{p}_{i}=e E_{i}+e B \epsilon_{i j} \dot{x}_{j}
$$

where the parameter $\theta=k / m^{2}$ measures the non-commutativity of $x_{1}$ and $x_{2}$, and we have introduced the effective mass

$$
m^{*}=m(1-e \theta B) .
$$

The novel features, crucial for physical applications, are twofold: Firstly, the relation between velocity and momentum contains an "anomalous velocity term" $-e m \theta \epsilon_{i j} E_{j}$, so that $\dot{x}_{i}$ and $p_{i}$ are not in general parallel. The second one is the interplay between the exotic structure and the magnetic field, yielding the effective mass $m^{*}$ in (2.4).

Equations (2.3) do not derive from a configuration-space Lagrangian (but see Section 3). The 1-order "phase" (in fact "evolution space" [3]) formalism works, however, so that the equations of motion (2.3) come from varying the action defined by integrating the "Cartan" 1-form [3],

$$
\lambda=\left(p_{i}-A_{i}\right) d x_{i}-\frac{\vec{p}^{2}}{2 m} d t+\frac{\theta}{2} \epsilon_{i j} p_{i} d p_{j}
$$

along the lifted world-line $\widetilde{\gamma}$ in evolution space $T^{*} \mathbb{R}^{2} \times \mathbb{R}$. The exterior derivative, $\sigma=d \lambda$, of the Cartan form $\lambda$ provides us with a closed "Lagrange-Souriau" 2-form, which, however, cannot be separated canonically into a "symplectic" and a "Hamiltonian part" [3]. Thus more general procedures have to be adopted to build such a system and clarify their Hamiltonian structure. These aspects will be discussed in detail in the following Sections 3,4 and 5. Here we limit ourselves to notice that, in fact, when $m^{*} \neq 0,(2.3)$ is a Hamiltonian system,

$$
\dot{\xi}=\left\{H, \xi^{\alpha}\right\}, \quad \xi=\left(x_{j}, p_{i}\right),
$$

with Poisson brackets

$$
\left\{x_{1}, x_{2}\right\}=\frac{m}{m^{*}} \theta, \quad\left\{x_{i}, p_{j}\right\}=\frac{m}{m^{*}} \delta_{i j}, \quad\left\{p_{1}, p_{2}\right\}=\frac{m}{m^{*}} e B .
$$

A remarkable property is that for vanishing effective mass $m^{*}=0$, i.e., when the magnetic field takes the critical value

$$
B=\frac{1}{e \theta}
$$

the system becomes singular. Then "Faddeev-Jackiw" (alias symplectic) reduction yields an essentially two-dimensional, simple system, reminiscent of "Chern-Simons mechanics" [32]. The symplectic plane plays, simultaneously, the role of both configuration and phase space. The only motions are those which follow a generalized Hall law. 
Quantization of the reduced system yields, moreover, the "Laughlin" wave functions [33], which are the ground states in the Fractional Quantum Hall Effect (FQHE).

The relations (2.5) diverge as $m^{*} \rightarrow 0$, but after reduction we get, cf. (1.1),

$$
\left\{x_{1}, x_{2}\right\}=\frac{1}{e B}=\theta \text {. }
$$

The coordinates are, hence, non-commuting, and their commutator is determined by the magnetic field, fine-tuned to the parameter $\theta$.

Relation to another non-commutative mechanics. The exotic relations (2.5) are similar to those proposed (later) in [10],

$$
\left\{x_{i}, x_{j}\right\}=\theta \epsilon_{i j}, \quad\left\{x_{i}, p_{j}\right\}=\delta_{i j}, \quad\left\{p_{1}, p_{2}\right\}=e B,
$$

which look indeed simpler. Using the standard Hamiltonian $H=\frac{p^{2}}{2 m}+e V(x)$, the associated equations of motion read

$$
m x_{i}^{\prime}=p_{i}-e m \theta \epsilon_{i j} E_{j}, \quad p_{i}^{\prime}=e B \epsilon_{i j} \frac{p_{j}}{m}+e E_{i},
$$

where we noted "time" by $T$ and $(\cdot)^{\prime}=\frac{d}{d T}$.

A short calculation reveals, however, that

$$
\left\{x_{i},\left\{p_{1}, p_{2}\right\}\right\}_{\text {cycl }}=e \theta \epsilon_{i j} \partial_{j} B,
$$

so that the Jacobi identity is only satisfied if $B$ is a constant. In other words, the system (2.6) is only consistent for a constant magnetic field - which is an unphysical condition in general ${ }^{2}$.

The model (2.6) has another strange feature. Let us indeed assume that the magnetic field is radially symmetric, $B=B(r)$. One would then expect to have conserved angular momentum. For constant $B$, applying Noether's theorem to an infinitesimal rotation $\delta \xi_{i}=\epsilon_{i j} \xi_{j}$ yields indeed $\delta \xi_{i}=-\left\{j^{\mathrm{NP}}, \xi_{i}\right\}$, with

$$
j^{\mathrm{NP}}=\frac{1}{1-e \theta B} \underbrace{\left(\vec{x} \times \vec{p}+\frac{\theta}{2} \vec{p}^{2}+\frac{e B}{2} \vec{x}^{2}\right)}_{j} .
$$

This differs from the standard expression by the pre-factor $(1-e \theta B)^{-1}$. But what is even worse is that, for a radial but non constant magnetic field, (2.8) is not conserved:

$$
\frac{d j^{\mathrm{NP}}}{d T}=\frac{e \theta j}{(1-e \theta B)^{2}} \partial_{i} B x_{i}^{\prime},
$$

while $j$ in (2.1) is still conserved as it should.

Can the theory defined by (2.6) be extended to an arbitrary $B$ ?

Let us first assume that $B=$ const. s.t. $m^{*} \neq 0$, and let us redefine the time $e^{3}$, as

$$
T \rightarrow t=(1-e \theta B) T \Rightarrow \frac{d}{d T}=(1-e \theta B) \frac{d}{d t} .
$$

Then equations (2.7) are carried into the exotic equations, (2.3). When $B=$ const. s.t. $m^{*} \neq$ 0 , the two theories are therefore equivalent.

Remarkably, the time redefinition (2.9) actually extends the previous theory, since it carries it into the "exotic model", for which the Jacobi identity holds for any, not necessarily constant B. Thus, the transformation (2.9) (which is singular for $e B \theta=1$ ), removes the unphysical restriction to constant magnetic fields: (2.9) regularizes the system (2.6).

\footnotetext{
${ }^{2}$ For another role of the Jacobi identity in non-commutative mechanics, see [34].

${ }^{3}$ This was suggested to us by G. Marmo (private communication).
} 


\section{Acceleration-dependent Lagrangian in configuration space}

An independent and rather different approach was followed in [8].

We start again with a particle characterized by the two central charges

$$
m \quad \text { and } \quad \kappa=-m^{2} \theta
$$

of the exotic Galilei group. These charges appear in the following Lie-brackets (represented by Poisson-brackets $(\mathrm{PBs})$ ) between the translation generators $P_{i}$ and the boost generators $K_{i}$

$$
\left\{P_{i}, K_{j}\right\}=m \delta_{i j}, \quad\left\{K_{i}, K_{j}\right\}=-m^{2} \theta \epsilon_{i j} .
$$

In order to find a configuration space Lagrangian whose Noether charges for boosts satisfy (3.1) we must add the second time derivative of the coordinates $\ddot{x}_{i}$ to the usual variables $x_{i}$ and $\dot{x}_{i}$. As shown in [8] the most general one-particle Lagrangian, which is at most linearly dependent on $\ddot{x}_{i}$, leading to the Euler-Lagrange equations of motion which are covariant w.r.t. the planar Galilei group, is given, up to gauge transformations, by

$$
\mathcal{L}=\frac{m}{2} \dot{x}_{i}^{2}+\frac{m^{2} \theta}{2} \epsilon_{i j} \dot{x}_{i} \ddot{x}_{j}
$$

Introducing the Lagrange multipliers $p_{i}$ and adding $p_{i}\left(\dot{x}_{i}-y_{i}\right)$ to $(3.2)$ yields

$$
\mathcal{L}=p_{i} \dot{x}_{i}+\frac{m^{2} \theta}{2} \epsilon_{i j} y_{i} \dot{y}_{j}-H(\vec{y}, \vec{p})
$$

with

$$
H(\vec{y}, \vec{p})=y_{i} p_{i}-\frac{m}{2} y_{i}^{2} .
$$

(3.3) describes a constrained system, because we have

$$
\frac{\partial \mathcal{L}}{\partial \dot{y}_{i}}=-\frac{m^{2} \theta}{2} \epsilon_{i j} y_{j}
$$

Therefore the PBs, obtained by means of the Faddeev-Jackiw procedure, take a non-standard form

$$
\left\{x_{i}, p_{j}\right\}=\delta_{i j}, \quad\left\{y_{i}, y_{j}\right\}=-\frac{1}{m^{2} \theta} \epsilon_{i j} .
$$

All other PBs vanish.

For the conserved boost generator we obtain

$$
K_{i}=-m x_{i}+p_{i} t-m^{2} \theta \epsilon_{i j} y_{j}
$$

and therefore, due to (3.4), the PB resp. commutator of two boosts is nonvanishing

$$
\left\{K_{i}, K_{j}\right\}=-m^{2} \theta \epsilon_{i j} .
$$

The Lagrangian (3.3) shows that the phase space is 6-dimensional. In order to split off two internal degrees of freedom, we have to look for a Galilean invariant decomposition of the 6-dim phase space into two dynamically independent parts: a 4-dim external and a 2-dim internal part. This decomposition is achieved by the transformation $[8,19](\vec{x}, \vec{p}, \vec{y}) \rightarrow(\vec{X}, \vec{p}, \vec{Q})$ with

$$
y_{i}=\frac{p_{i}}{m}+\frac{Q_{i}}{m \theta} \quad \text { and } \quad x_{i}=X_{i}-\epsilon_{i j} Q_{j},
$$


leading to the following decomposition of the Lagrangian (3.3)

$$
\mathcal{L}=\mathcal{L}_{\text {ext }}+\mathcal{L}_{\text {int }}
$$

with

$$
\mathcal{L}_{\text {ext }}=p_{i} \dot{X}_{i}+\frac{\theta}{2} \epsilon_{i j} p_{i} \dot{p}_{j}-\frac{p_{i}^{2}}{2 m} \quad \text { and } \quad \mathcal{L}_{\text {int }}=\frac{1}{2 m \theta^{2}} Q_{i}^{2}+\frac{1}{2 \theta} \epsilon_{i j} Q_{i} \dot{Q}_{j}
$$

From (3.6) and the PBs (3.4) it now follows that the new coordinates $X_{i}$ are noncommutative

$$
\left\{X_{i}, X_{j}\right\}=\theta \epsilon_{i j}
$$

The remaining nonvanishing PBs are

$$
\left\{X_{i}, p_{j}\right\}=\delta_{i j}, \quad\left\{Q_{i}, Q_{j}\right\}=-\theta \epsilon_{i j} .
$$

Conclusion. The particle Lagrangian (3.2) containing $\ddot{x}_{i}$ leads to a nonvanishing commutator of two boosts. But in order to obtain noncommutative coordinates we are forced to decompose the 6-dim phase space in a Galilean invariant manner into two dynamically independent 4-dim external and 2-dim internal phase spaces.

Relation of the DH and LSZ models. The relation of the "minimal" and the configuration-space models of DH [9] and of L.S.Z. [8] respectively, has been studied in [19]. Introducing the coordinates $X_{i}, Q_{i}$, and $p_{i}$ on 6-dimensional phase space according to (3.6) allows us to present the symplectic structure and the Hamiltonian associated with (3.2) as

$$
\begin{aligned}
& \Omega=d p_{i} \wedge d X_{i}+\frac{\theta}{2} \varepsilon_{i j} d p_{i} \wedge d p_{j}+\frac{1}{2 \theta} \varepsilon_{i j} d Q_{i} \wedge d Q_{j}, \\
& H=\frac{\vec{p}^{2}}{2 m}-\frac{1}{2 m \theta^{2}} \vec{Q}^{2} .
\end{aligned}
$$

Thus, the model of L.S.Z. is decomposed into the DH theory, augmented with a two-dimensional internal space, and a negative zero point of the Hamiltonian. Note that the "external" and "internal" phase spaces are "almost" independent: the only effect of internal motion is indeed through the length of the internal vector, $|\vec{Q}|$.

Generalization of (3.2). If we add to (3.2) a term $f\left(\ddot{x}_{i}^{2}\right)$, the obtained Lagrangian is the most general one involving, in a Galilean quasi-invariant manner, the variables $x_{i}, \dot{x}_{i}$ and $\ddot{x}_{i}$.

Then one can show

i) the $\mathrm{PB}$ of the two boosts (3.5) will not change,

ii) the new 8-dim phase space may be decomposed again in a Galilean invariant manner into two dynamically independent 4-dim parts, an external and an internal one.

\section{Commutative - versus noncommutative plane.}

The subalgebra of the Galilean algebra containing only translations and boosts is given in the cases of, respectively, their one- or two-fold central extensions by

$$
\begin{array}{ll}
\frac{\text { one-fold centrally extended }}{\left\{p_{i}, K_{j}\right\}=m \delta_{i j},} & \frac{\text { two-fold centrally extended }}{\left\{p_{i}^{\prime}, K_{j}^{\prime}\right\}=m \delta_{i j},} \\
\left\{p_{i}, p_{j}\right\}=0, & \left\{p_{i}^{\prime}, p_{j}^{\prime}\right\}=0, \\
\left\{K_{i}, K_{j}\right\}=0, & \left\{K_{i}^{\prime}, K_{j}^{\prime}\right\}=-m^{2} \theta \epsilon_{i j} .
\end{array}
$$

Obviously both are related by the transformations

$$
K_{i}^{\prime}=K_{i}-\frac{m \theta}{2} \epsilon_{i j} p_{j}, \quad p_{i}^{\prime}=p_{i}
$$


To this corresponds the following point transformation between noncommutative coordinates $X_{i}$ and commutative ones $q_{i}$

$$
X_{i}=q_{i}-\frac{\theta}{2} \epsilon_{i j} p_{j}
$$

as can be read off immediately from the form of $\mathcal{L}_{\text {ext }}$ in (3.7).

Now the question arises: What to use in physics, the commutative or the non-commutative plane?

Answer. For free particles both possibilities are equivalent. But in the case of a nontrivial interaction one has to use the commutative (noncommutative) plane, if a local potential or gauge interaction is given in terms of $q_{i}\left(X_{i}\right)$.

It is worth mentioning that the acceleration-dependent model presented in this Section can be related to radiation damping [35].

\section{General form of noncommutative mechanics}

Up to now noncommutativity has been described by a constant $\theta$ in the PB (3.8). But it is possible to get $\theta$ as a function of $\vec{X}$ and $\vec{p}$ if one considers external Lagrangians more general than (3.7).

To do this consider a very general class of Lagrangians given by

$$
\mathcal{L}=p_{i} \dot{X}_{i}+\tilde{A}_{i}(\vec{X}, \vec{p}) \dot{p}_{i}-H(\vec{p}, \vec{X})
$$

leading to the PBs

$$
\left\{X_{i}, X_{j}\right\} \sim \epsilon_{i j} \tilde{B}, \quad \tilde{B}=\epsilon_{k \ell} \partial_{p_{k}} \tilde{A}_{\ell}(\vec{X}, \vec{p})
$$

with

$$
\left\{P_{i}, P_{j}\right\}=0 .
$$

We dispense with the reproduction of the more complicated form of the PBs for the phase space coordinates $\left(X_{i}, p_{j}\right)$. Again by the point transformation

$$
X_{i} \rightarrow q_{i}=X_{i}-\tilde{A}_{i}(\vec{X}, \vec{p})
$$

we obtain commuting coordinates $q_{i}$ as follows from

$$
p_{i} \dot{X}_{i}+\tilde{A}_{i} \dot{p}_{i}=p_{i} \dot{q}_{i}+\frac{d}{d t}\left(\tilde{A}_{i} p_{i}\right) .
$$

Examples:

i)

$$
\tilde{A}_{i}=f\left(p^{2}\right)(\vec{X} \cdot \vec{p}) p_{i}
$$

leading to the PBs of the phase space variables

$$
\begin{aligned}
& \left\{X_{i}, X_{j}\right\}=\frac{f\left(p^{2}\right)}{1-p^{2} f\left(p^{2}\right)} \epsilon_{i j} L, \quad L=\epsilon_{k \ell} X_{k} p_{\ell}, \\
& \left\{X_{i}, p_{j}\right\}=\delta_{i j}+\frac{f\left(p^{2}\right)}{1-p^{2} f\left(p^{2}\right)} p_{i} p_{j} .
\end{aligned}
$$


A particular example is given by

$$
f\left(p^{2}\right)=\frac{\theta}{1+p^{2} \theta} \quad \text { and therefore } \quad \frac{f}{1-p^{2} f}=\theta .
$$

This gives exactly Snyder's NC-algebra, presented in 1947 [36].

Another case, defined by

$$
f\left(p^{2}\right)=\frac{2}{p^{2}}
$$

can be related to a deformed Galilei algebra (to be discussed in the next section).

ii)

$$
\tilde{A}_{i}=\tilde{A}_{i}(\vec{p})
$$

leading to the PBs

$$
\left\{X_{i}, X_{j}\right\}=\epsilon_{i j} \tilde{B}(\vec{p}), \quad\left\{X_{i}, p_{j}\right\}=\delta_{i j} .
$$

$\tilde{B}$ is the Berry curvature for the semiclassical dynamics of electrons in condensed matter to be discussed in Section 11.

We may generalize (4.1) to the most general 1st-order Lagrangian

$$
\mathcal{L}=\left(p_{i}+A_{i}(\vec{X}, \vec{p})\right) \dot{X}_{i}+\tilde{A}_{i}(\vec{X}, \vec{p}) \dot{p}_{i}-H(\vec{p}, \vec{X}) .
$$

Here $A_{i}(\vec{X})$ describes standard electromagnetic interaction (cp. Section 6, and Section 11 for the 3 dimensional case, respectively). A particular case of a $\vec{p}$-dependent $A_{i}$ has been considered in [37].

\section{Lagrangian realization of the $\tilde{\boldsymbol{k}}$-deformed Galilei algebra as a symmetry algebra}

In 1991 Lukierski, Nowicki, Ruegg and Tolstoy invented the $k$-deformed Poincaré algebra [38], which later found applications, e.g., in Quantum Gravity [39]. By rescaling the Poincaré generators and the deformation parameter $k$, the corresponding nonrelativisitic limit, the $\tilde{k}$-deformed Galilei algebra, has been derived by Giller et al. [40] and, in a different basis, by Azcarraga et al. [41]. In this Section we will describe a Lagrangian realization of the latter.

Again we look at the classical Lagrangian (4.1) specified by (4.2) and (4.5) together with the following choice of the Hamiltonian

$$
H=\tilde{k} \ln \left(p^{2} / 2\right) \text {. }
$$

According to (4.3), (4.4) we obtain the PBs

$$
\left\{X_{i}, X_{j}\right\}=-\frac{2}{p^{2}} \epsilon_{i j} L \quad \text { and } \quad\left\{X_{i}, p_{j}\right\}=\delta_{i j}-\frac{2}{p^{2}} p_{i} p_{j}
$$

which lead, together with the Hamiltonian (5.1), to the equations of motion

$$
\dot{p}_{i}=0 \quad \text { and } \quad \dot{X}_{i}=-\frac{2 \tilde{k}}{p^{2}} p_{i}
$$


Then, we may define the "pseudo-boosts" $K_{i}$

$$
K_{i}=p_{i} t+\frac{p^{2}}{2 \tilde{k}} X_{i}
$$

which are conserved. They satisfy, together with $p_{i}$ and $H$, the PB-algebra

$$
\left\{K_{i}, p_{j}\right\}=\frac{\delta_{i j}}{2 \tilde{k}} p^{2}-\frac{p_{i} p_{j}}{\tilde{k}}, \quad\left\{K_{i}, H\right\}=-p_{i}, \quad\left\{K_{i}, K_{j}\right\}=0 .
$$

Together with the standard algebra of translations (represented by $p_{i}$ and $H$ ) and rotations (represented by $L$ ) the relations (5.2), build the $\tilde{k}$-deformed Galilei algebra derived in [41].

The limit $\tilde{k} \rightarrow \infty$ leads to a divergent Hamiltonian (5.1). Therefore, the $\tilde{k}$-deformation does not have a standard "no-deformation limit".

\section{Physical origin of the exotic structure}

A free relativistic "elementary" particle in the plane corresponds to a unitary representation of the planar Lorentz group $O(2,1)$ [17]. These representations are in turn associated with the coadjoint orbits of $S O(2,1)$, endowed with their canonical symplectic structures, interpreted by Souriau as classical phase spaces [3]. Applied to the planar Lorentz group, the procedure yields the relativistic model $[17,18]$

$$
\begin{aligned}
& \Omega_{\mathrm{rel}}=d p_{\alpha} \wedge d x^{\alpha}+\frac{s}{2} \epsilon^{\alpha \beta \gamma} \frac{p_{\alpha} d p_{\beta} \wedge d p_{\gamma}}{\left(p^{2}\right)^{3 / 2}}, \\
& H_{\mathrm{rel}}=\frac{1}{2 m}\left(p^{2}-m^{2} c^{2}\right) .
\end{aligned}
$$

The $p$-dependent contribution looks like a "magnetic monopole in momentum space" (cf. (11.10) below).

As pointed out by Jackiw and Nair [16], the free exotic model can be recovered considering a tricky non-relativistic limit, namely

$$
s / c^{2} \rightarrow \kappa=m^{2} \theta .
$$

The two-form $\left.\Omega_{\text {rel }}\right|_{H_{\text {rel }}=0}$ goes indeed over into the exotic symplectic form. Intuitively, the exotic structure can be viewed as a "non-relativistic shadow" of relativistic spin.

The exotic Galilei group can itself be derived from the planar Poincaré group by "JackiwNair" contraction [16]. One starts with the planar Lorentz generators,

$$
\left\{J^{\alpha}, J^{\beta}\right\}=\epsilon^{\alpha \beta \gamma} J_{\gamma} .
$$

For the classical system

$$
J_{\mu}=\epsilon_{\mu \nu \rho} x^{\nu} p^{\rho}+s \frac{p_{\mu}}{\sqrt{p^{2}}} .
$$

A non-relativistic boost is the "JN" limit of a suitable Lorentz transformation,

$$
\frac{1}{c} \epsilon_{i j} J^{j} \rightarrow m x_{i}-p_{i} t+m \theta \epsilon_{i j} p_{j}=-K_{i},
$$

and the exotic relation is recovered,

$$
\left\{K_{1}, K_{2}\right\}=J_{0} / c^{2} \rightarrow \frac{s}{c^{2}}=\kappa .
$$


The angular momentum is in turn

$$
J_{0}=\vec{x} \times \vec{p}+s+\frac{s}{m^{2} c^{2}} \vec{p}^{2} \rightarrow \vec{x} \times \vec{p}+\frac{\theta}{2} \vec{p}^{2}=j .
$$

whereas the divergent term $s=\kappa c^{2}$ has to be removed by hand.

It is worth mentioning that the "Jackiw-Nair limit" of a relativistic particle with torsion [42] provides us with the L.S.Z. model [19], and a similar procedure yields the so-called "NewtonHooke" system [43]. Applied to the infinite-component Majorana-type anyon equations [17, 18] yields, furthermore, a first-order infinite-component "Lévy-Leblond type" system with exotic Galilean symmetry [20].

About anyons constructed from orbits, see also [44].

\section{Anomalous coupling of anyons}

It has been suggested [45] that a classical, relativistic anyon in an electromagnetic field should be described by the equations

$$
\begin{array}{ll}
m \frac{d x^{\alpha}}{d \tau}=p^{\alpha} & \text { (velocity-momentum), } \\
\frac{d p^{\alpha}}{d \tau}=\frac{e}{m} F^{\alpha \beta} p_{\beta} & \text { (Lorentz equation), }
\end{array}
$$

$\alpha, \beta, \ldots=0,1,2$ and $\tau$ denotes the proper time. These equations are Hamiltonian, with symplectic form and Hamilton's function

$$
\begin{aligned}
& \Omega=\Omega_{\mathrm{rel}}+\frac{1}{2} e F_{\alpha \beta} d x^{\alpha} \wedge d x^{\beta}, \\
& H=H_{\mathrm{rel}}+\frac{e s}{2 m \sqrt{p^{2}}} \epsilon_{\alpha \beta \gamma} F^{\alpha \beta} p^{\gamma},
\end{aligned}
$$

respectively. Let us observe that the second, non-minimal term in the Hamiltonian is dictated by the required form of the velocity relation in (7.1).

The model of [45] has gyromagnetic ratio $g=2$, and some theoreticians have long believed $[45,46]$ that this is indeed the "correct" $g$ value of anyons. Experimental evidence shows, however, that in the Fractional Quantum Hall Effect, for example, the measured value of $g$ is approximately zero [47]. Is it possible to construct an "anomalous" model with $g \neq 2$ ? The answer is affirmative [48], as we now explain.

Planar spin has to satisfy the relation $S_{\alpha \beta} p^{\beta}=0$. The spin tensor has, therefore, the form

$$
S_{\alpha \beta}=\frac{s}{\sqrt{p^{2}}} \epsilon_{\alpha \beta \gamma} p^{\gamma}
$$

Introducing the shorthand $-F_{\alpha \beta} S^{\alpha \beta}=F \cdot S$, the Hamiltonian (7.2) is presented as

$$
H^{\mathrm{CNP}}=\frac{1}{2 m}\left(p^{2}-M^{2} c^{2}\right) \quad \text { where } \quad M^{2}=m^{2}+\frac{e}{c^{2}} F \cdot S .
$$

Let us observe that the "mass" $M$ depends here on spin-field coupling. Our clue for generalizing this model has been the formula put forward by Duval $[49,50]$ : let us posit, instead of (7.3), the mass formula

$$
M^{2}=m^{2}+\frac{g}{2} \frac{e}{c^{2}} F \cdot S,
$$


where $g$ is an arbitrary real constant. Then consistent equations of motion are obtained for any $g$, namely

$$
\begin{aligned}
& D \frac{d x^{\alpha}}{d \tau}=G \frac{p^{\alpha}}{M}+(g-2) \frac{e s}{4 M^{2}} \epsilon^{\alpha \beta \gamma} F_{\beta \gamma}, \\
& \frac{d p^{\alpha}}{d \tau}=\frac{e}{m} F^{\alpha \beta} p_{\beta},
\end{aligned}
$$

where the coefficients denote the complicated, field-dependent expressions

$$
D=1+\frac{e F \cdot S}{2 M^{2} c^{2}}, \quad G=1+\frac{g}{2} \frac{e F \cdot S}{2 M^{2} c^{2}} .
$$

Choosing $g=2$, the generalized model plainly reduces to equation (7.1), proposed in [45]. We stress, however, that no physical principle requires that the second, "anomalous" term should vanish in (7.4). $g=2$ is not a physical necessity therefore: a perfectly consistent model is obtained for any $g$, as it has been advocated a long time ago $[49,50]$.

Non-relativistic anyon with anomalous coupling. We can now consider the "JackiwNair" non-relativistic limit of the above relativistic model. This provides us, for any $g$, with the Lorentz equation (7.5), supplemented with

$$
\left(M_{g} D\right) \dot{x}_{i}=G p_{i}-\left(1-\frac{g}{2}\right) e M_{g} \theta \epsilon_{i j} E_{j}
$$

where

$$
\left.M_{g}=m(\sqrt{1-g \theta e B}), \quad D=(1-(g+1) \theta e B), \quad G=(1-(3 g / 2)) \theta e B\right) .
$$

- It is a most important fact that, for any $g \neq 2$, the only consistent motions follow a generalized Hall law, whenever the field takes either of the critical values

$$
B=\frac{1}{1+g} \frac{1}{e \theta} \quad \text { or } \quad \frac{2}{3 g} \frac{1}{e \theta} .
$$

One can indeed show that, for any $g \neq 2$, the models can be transformed into each other by a suitable redefinition. For $g=0$ the equations become identically satisfied. See [48] for details.

- In particular, for $g=0$ the minimal exotic model of [9] is recovered. The latter is, hence, not the NR limit of the model of [45] (7.1) [which has $g=2$, as said]. The experimental evidence [47] is, hence, a strong argument in favor of the minimal model of [9].

- $g=2$ is the only case when the velocity and the momentum are parallel. This is, however, not required by any first principle.

Having an anomalous velocity relation seems to be unusual in high-energy physics; it is, however, a well accepted requirement in condensed matter physics, as explained in Section 11.

Let us mention that relativistic anyons can be described, at the field theoretical level, by infinite-component fields of the Majorana-Dirac type [18]. Coupling them to an external gauge field is a major unsolved problem. Partial results can be obtained in the non-relativistic case [51].

\section{Two ways of introducing electromagnetic interactions}

In this section we will show that Souriau's coupling prescription (2.2) is not the only possibility to introduce electromagnetic (e.m.) interaction into the Lagrangian $\mathcal{L}_{\text {ext }}(3.7)$.

In the commutative case we have the principle of minimal e.m. coupling

$$
p_{i} \dot{X}_{i}-\frac{p_{i}^{2}}{2 m} \rightarrow\left(p_{i}+e A_{i}(\vec{X}, t)\right) \dot{X}_{i}-\frac{p_{i}^{2}}{2 m}+e A_{0}(\vec{X}, t),
$$


called the minimal additon rule, which is equivalent, due to the point transformation $p_{i} \rightarrow$ $p_{i}-e A_{i}$, to the minimal substitution rule [52],

$$
p_{i} \dot{X}_{i}-\frac{p_{i}^{2}}{2 m} \rightarrow p_{i} \dot{X}_{i}-\frac{\left(p_{i}-e A_{i}\right)^{2}}{2 m}+e A_{0}(\vec{X}, t) .
$$

In the noncommutative case the equivalence of minimal addition and minimal substitution rule is not valid. Therefore we have to consider two different ways of introducing the minimal e.m. coupling:

Minimal addition (Duval-Horvathy [9], called DH-model)

$$
\mathcal{L} \rightarrow \mathcal{L}_{\text {e.m. }}=\mathcal{L}+e\left(A_{i} \dot{X}_{i}+A_{0}\right),
$$

which, as usual, is quasi-invariant w.r.t. standard gauge transformations

$$
A_{\mu}(\vec{X}, t) \rightarrow A_{\mu}(\vec{X}, t)+\partial_{\mu} \Lambda(\vec{X}, t) .
$$

Obviously the minimal addition rule (8.1) is equivalent to Souriau's prescription (2.2).

Minimal substitution (Lukierski-Stichel-Zakrzewski [52], called L.S.Z. model) ${ }^{4}$

$$
H=\frac{p_{i}^{2}}{2 m} \rightarrow H_{\mathrm{e} . \mathrm{m} .}=\frac{\left(p_{i}-e \hat{A}_{i}\right)^{2}}{2 m}-e \hat{A}_{0} .
$$

The corresponding Lagrangian is quasi-invariant w.r.t. generalized gauge transformations, given in infinitesimal form by

$$
\delta \hat{A}_{\mu}(\vec{X}, t)=\hat{A}_{\mu}^{\prime}(\vec{X}+\delta \vec{X}, t)-\hat{A}_{\mu}(\vec{X}, t)=\partial_{\mu} \Lambda(\vec{X}, t),
$$

with

$$
\delta X_{i}=-e \theta \epsilon_{i j} \partial_{j} \Lambda
$$

and supplemented by

$$
\delta p_{i}=e \partial_{i} \Lambda
$$

Note that the coordinate transformations (8.2) are area preserving.

It turns out that both models are related to each other by a noncanonical transformation of phase space variables supplemented by a classical Seiberg-Witten transformation of the corresponding gauge potentials:

If we denote the phase space variables and potentials for

- the DH-model by $\left(\vec{\eta}, \overrightarrow{\mathcal{P}}, A_{\mu}\right)$,

- the L.S.Z.-model by $\left(\vec{X}, \vec{p}, \hat{A}_{\mu}\right)$,

then we find the relations

$$
\begin{aligned}
& \eta_{i}(\vec{X}, t)=X_{i}+e \theta \epsilon_{i j} \hat{A}_{j}(\vec{X}, t), \\
& \mathcal{P}_{i}=p_{i}-e \hat{A}_{i}(\vec{X}, t)
\end{aligned}
$$

with the corresponding field strengths related by

$$
\hat{F}_{\mu \nu}(\vec{X}, t)=\frac{F_{\mu \nu}(\vec{\eta}, t)}{1-e \theta B(\vec{\eta}, t)} .
$$

\footnotetext{
${ }^{4}$ In this model the gauge fields carry a "hat" in order to distinguish them from the corresponding quantities in the DH-model.
} 
The Seiberg-Witten transformation between the resp. gauge fields is more involved, and will not be reproduced here (for details cp. [52]).

These results lead to an interesting by-product: Consider the PBs of coordinates in both models, given by

$$
\left\{\eta_{i}, \eta_{j}\right\}=\frac{\theta \epsilon_{i j}}{1-e \theta B(\vec{\eta}, t)} \quad \text { and } \quad\left\{X_{i}, X_{j}\right\}=\theta \epsilon_{i j}
$$

Then the foregoing results implicitly give the coordinate transformation between a model with a constant noncommutativity parameter $m^{2} \theta$ and one with arbitrary coordinate-dependent noncommutativity function $m^{2} \theta(\vec{X}, t)$ (this result has been rediscovered in [53]).

Now the question arises, which of both models has to be used for physical applications? Let us look at one example, the Quantum Hall effect. As already shown in Section 2 in the case of the DH-model [9] the Hall law,

$$
\dot{X}_{i}=\epsilon_{i j} \frac{E_{j}}{B},
$$

is valid at the critical magnetic field

$$
B_{\text {crit }}=(e \theta)^{-1} \text {. }
$$

Then it follows from the field transformation law (8.3) that, for the L.S.Z.-model, the Hall law is valid in the limit of large e.m. fields. In order to see this in more detail we have to consider the equations of motion for the L.S.Z.-model formulated in terms of the gauge-invariant phase space

variables $\vec{\eta}$ and $\overrightarrow{\mathcal{P}}$. For that, we use the equations of motion (2.3) for the DH model written in terms of $\eta_{i}$ and $\mathcal{P}_{i}$, transform the e.m. fields according to (8.3) and we obtain $(e=1, m=1)$

$$
\begin{aligned}
& \dot{\eta}_{i}=(1+\theta \hat{B}) \mathcal{P}_{i}-\theta \epsilon_{i j} \hat{E}_{j}, \\
& \dot{\mathcal{P}}_{i}=\hat{B} \epsilon_{i j} \mathcal{P}_{j}+\hat{E}_{i} .
\end{aligned}
$$

For the particular case of homogeneous e.m. fields we obtain finally

$$
\ddot{\eta}_{i}=\hat{B} \epsilon_{i j} \dot{\eta}_{j}+\hat{E}_{i}
$$

leading to the Hall law (8.5) in the high field limit.

Note that (8.7) has the same functional form as in the commutative case.

Another point of view is presented in [54].

\section{Supersymmetry}

In the following, we supersymmetrize the e.m. coupling models treated in the last section. To do that we follow the treatment in Section 3 of [55]. For that, we consider standard $N=2$ SUSY characterized by

$$
H=\frac{i}{2}\{Q, \bar{Q}\}
$$

and

$$
\{Q, Q\}=\{\bar{Q}, \bar{Q}\}=0 .
$$

In order to construct the supercharge $Q$, satisfying (9.1), we start with the common structure of the bosonic Hamiltonian $H_{b}$ for both models $(e=1, m=1)$

$$
H_{b}=\frac{1}{2}\left(\mathcal{P}_{i}^{2}+W_{i}^{2}(\vec{X})\right)
$$


with

$$
\mathcal{P}_{i}=p_{i} \quad \text { for the DH-model }
$$

and

$$
\mathcal{P}_{i}=p_{i}-A_{i} \quad \text { for the L.S.Z.-model. }
$$

Note that, in accordance with the quantized form of (9.1), the potential term in (9.3) is chosen to be positive

$$
A_{0}=-\frac{1}{2} W_{i}^{2} .
$$

In order to add to (9.3) its fermionic superpartner, we supplement the bosonic phase space variables with fermionic coordinates $\psi_{i}\left(\bar{\psi}_{i}\right)$ satisfying canonical PBs

$$
\left\{\psi_{i}, \bar{\psi}_{j}\right\}=-i \delta_{i j} .
$$

Now we assume

$$
Q=i\left(\mathcal{P}_{i}+i W_{i}\right) \psi_{i}
$$

such that (9.3) is valid. But now the relations (9.2) are fulfilled only if the following two conditions are satisfied:

$$
\left\{\mathcal{P}_{i}, \mathcal{P}_{j}\right\}=\left\{W_{i}, W_{j}\right\}
$$

and

$$
\left\{\mathcal{P}_{i}, W_{j}\right\}=\left\{\mathcal{P}_{j}, W_{i}\right\}
$$

It can be shown that (9.6) is satisfied automatically in both models, whereas (9.5) fixes the magnetic field in terms of $W_{i}$ (same form for both models):

$$
B=\frac{\theta}{2} \epsilon_{i j} \epsilon_{k \ell} \partial_{k} W_{i} \partial_{\ell} W_{j}
$$

The connection between B-field (9.7) and electric potential $A_{0}$ (9.4) takes a simple form in the case of rotational invariance. From

$$
W_{i}(\vec{X})=\partial_{i} W(r)
$$

we obtain

$$
A_{0}(r)=-\frac{1}{2}\left(W^{\prime}(r)\right)^{2}
$$

and

$$
B(r)=-\frac{\theta}{r} A_{0}^{\prime}(r)
$$

As an example, consider the harmonic oscillator. Then

$$
A_{0}=-\frac{\omega^{2}}{2} r^{2}
$$

and we obtain a homogeneous $B$-field of strength

$$
B=\theta \omega^{2} .
$$

The supersymmetric extension of the DH model, and of anyons, have also been studied in [56] and in [57], respectively. 


\section{Galilean symmetry in Moyal field theory}

As we mentioned already, the physical explanation of the Fractional Quantum Hall Effect (FQHE) relies on the dynamics of quasiparticles which carry both an electric and a magnetic charge [33]. In the field theory context, these quasiparticles arise as charged vortex solutions of the coupled field equations. The phenomenologically preferred theory of Zhang et al. [58] is Galilei invariant; the Galilean boost commute for these models, though. Does there exist a field theoretical model with "exotic" Galilean symmetry? The answer is yes, if we consider Moyal field theory $[14,59]$. Here one considers the usually-looking Lagrangian

$$
L=i \bar{\psi} D_{t} \psi-\frac{1}{2}|\vec{D} \psi|^{2}+\kappa\left(\frac{1}{2} \epsilon_{i j} \partial_{t} A_{i} A_{j}+A_{t} B\right)
$$

but where the covariant derivative and the field strength,

$$
\begin{aligned}
& D_{\mu} \psi=\partial_{\mu} \psi-i e A_{\mu} \star \psi, \\
& F_{\mu \nu}=\partial_{\mu} A_{\nu}-\partial_{\nu} A_{\mu}-i e\left(A_{\mu} \star A_{\nu}-A_{\nu} \star A_{\mu}\right),
\end{aligned}
$$

respectively, involve the Moyal "star" product, associated with the parameter $\theta$,

$$
(f \star g)\left(x_{1}, x_{2}\right)=\left.\exp \left(i \frac{\theta}{2}\left(\partial_{x_{1}} \partial_{y_{2}}-\partial_{x_{2}} \partial_{y_{1}}\right)\right) f\left(x_{1}, x_{2}\right) g\left(y_{1}, y_{2}\right)\right|_{\vec{x}=\vec{y}} .
$$

Here the matter field $\psi$ is in the fundamental representation of the gauge group $U(1)_{*}$ i.e., $A_{\mu}$ acts from the left. The associated field equations look formally as in the commutative case,

$$
\begin{aligned}
& i D_{t} \psi+\frac{1}{2} \vec{D}^{2} \psi=0, \\
& \kappa E_{i}-e \epsilon_{i k} j_{k}^{l}=0, \\
& \kappa B+e \rho^{l}=0,
\end{aligned}
$$

where $B=\epsilon_{i j} F_{i j}, E_{i}=F_{i 0}$. Note, however, that $\rho^{l}$ and $\vec{j}^{l}$ denote here the left density and left current, respectively,

$$
\rho^{l}=\psi \star \bar{\psi}, \quad \vec{j}^{l}=\frac{1}{2 i}(\vec{D} \psi \star \bar{\psi}-\psi \star(\overline{\vec{D} \psi})) .
$$

These theories admit static, finite-energy vortex solutions [59] which generalize those found before in ordinary CS theory [58, 60].

Are these theories Galilean invariant? At first sight, the answer seems to be negative, and it has been indeed a widely shared view that Moyal field theory is inconsistent with Galilean symmetry. The situation is more subtle, however. The conventional infinitesimal implementation of a Galilean boost,

$$
\delta^{0} B=-t \vec{b} \cdot \vec{\nabla} B \quad \text { but } \quad \delta^{0} \rho^{l}=-\frac{\theta}{2} \vec{b} \times \vec{\nabla} \rho^{l}-\vec{b} \cdot \vec{\nabla} \rho^{l} .
$$

is indeed broken, as the Gauss constraint (10.1) is not preserved. Galilean symmetry can be restored taking into account the Moyal structure [21], namely considering the antifundamental representation

$$
\delta^{r} \psi=\psi \star(i \vec{b} \cdot \vec{x})-t \vec{b} \cdot \vec{\nabla} \psi=(i \vec{b} \cdot \vec{x}) \psi+\frac{\theta}{2} \vec{b} \times \vec{\nabla} \psi-t \vec{b} \cdot \vec{\nabla} \psi
$$


Observing that

$$
\delta^{r} \psi=\delta^{0} \psi+\frac{\theta}{2} \vec{b} \times \vec{\nabla} \psi
$$

we find that the $\theta$-terms cancel in $\delta^{r} \rho^{l}$, leaving us with the homogeneous transformation law

$$
\delta^{r} \rho^{l}=-t \vec{b} \cdot \vec{\nabla} \rho^{l} .
$$

Putting $\delta^{r} A_{\mu}=\delta^{0} A_{\mu}$, so that $\delta^{r} B=\delta^{0} B$, the Gauss constraint (10.1) is right-invariant, as are all the remaining equations. The associated boost generator, calculated using the Noether theorem, reads

$$
\vec{K}^{r}=t \vec{P}-\int \vec{x} \rho^{r} d^{2} \vec{x}
$$

where

$$
P_{i}=\int \frac{1}{2 i}\left(\bar{\psi} \partial_{i} \psi-\left(\overline{\partial_{i} \psi}\right) \psi\right) d^{2} \vec{x}-\frac{\kappa}{2} \int \epsilon_{j k} A_{k} \partial_{i} A_{j} d^{2} \vec{x}
$$

is the conserved momentum. The conservation of (10.2) can also be checked directly, using the continuity equation satisfied by the right density, $\rho^{r}=\bar{\psi} \star \psi$. At last, the boost components have the exotic commutation relation

$$
\left\{K_{i}, K_{j}\right\}=\epsilon_{i j} k, \quad k \equiv-\theta \int|\psi|^{2} d^{2} x
$$

Let us note, in conclusion, that Galilean symmetry as established here makes it possible to produce moving vortices by boosting the static solutions constructed in [59], see [61].

\section{Noncommutativity in 3 dimensions: the semiclassical Bloch electron}

\subsection{The semiclassical model}

Around the same time and with no relation to the above developments, a very similar theory has arisen in condensed matter physics. For instance, applying a Berry-phase argument to a Bloch electron in a lattice, the standard semiclassical equations [62] are modified by new terms [22], generating purely quantum effects on the mean values of the electron's position and quasi-momentum $\vec{r}$ and $\vec{p}$, respectively, which add to the force due to the momentum gradient of the energy band dispersion relation $\epsilon_{n}(\vec{p})$ and to the external (for instance, Lorentz) forces. The semiclassical approach allows several applications and generalizations, both from the physical [22, 23, 24, 25, 13], and the mathematical [29, 63, 64, 65] side.

The clue is that the semiclassical model fits perfectly into Souriau's general framework [3] presented above. One starts with a "microscopic" Hamiltonian operator $\hat{H}[\hat{\vec{r}}, \hat{\vec{p}}, f(\hat{\vec{r}}, t)]$ for a particle (electron) for a periodic potential, which is adiabatically (in space-time) modified by a perturbation $f$ (possibly an external field). The position/momentum operators $\hat{\vec{r}}$ and $\hat{\vec{p}}$ satisfy the Heisenberg algebra, as usual. Moreover, for any constant $f$, the Hamiltonian $\hat{H}$ reduces to the usual one for a periodic crystal lattice.

The adiabatic features of the perturbation $f$ are expressed by the inequalities $l_{\text {latt }} \ll l_{w p} \ll$ $l_{\text {mod }}$, among the lattice constant length $l_{\text {latt }}$, the wave-packet dispersion length $l_{w p}$ and the modulation wave-length $l_{\text {mod }}$. Furthermore, the characteristic time scale $\hbar / \Delta E_{\text {gap }}$ must be much smaller than the typical time-scale of variations of $f$. 
The first order truncation of the Hamiltonian around the instantaneous mean position $\vec{r}_{c}$,

$$
\begin{aligned}
& \hat{H}[\hat{\vec{r}}, \hat{\vec{p}}, f(\hat{\vec{r}}, t)]=\hat{H}_{\left(\vec{r}_{c}, t\right)}+\hat{W}_{\left(\vec{r}_{c}, t\right)}, \\
& \hat{W}_{\left(\vec{r}_{c}, t\right)}=\frac{1}{2}\left[\partial_{f} \hat{H} \nabla_{\vec{r}_{c}} f\left(\vec{r}_{c}, t\right) \cdot\left(\hat{\vec{r}}-\vec{r}_{c}\right)+\text { h.c. }\right],
\end{aligned}
$$

defines a quasi-static Hamiltonian $\hat{H}_{\left(\vec{r}_{c}, t\right)}$, depending on the "slow" parameters $c=\left(\vec{r}_{c}, t\right) . \hat{H}_{\left(\vec{r}_{c}, t\right)}$ is periodic under $\vec{a}$-translations, and its eigenstates are Bloch the waves. The latter are defined, for any fixed time $t$ and $\vec{r}_{c}$, by

$$
\begin{array}{ll}
\hat{H}_{\left(\vec{r}_{c}, t\right)}\left|\psi_{\left(\vec{r}_{c}, t\right)}^{n, \vec{q}}\right\rangle=E_{\left(\vec{r}_{c}, t\right)}^{n, \vec{q}}\left|\psi_{\left(\vec{r}_{c}, t\right)}^{n, \vec{q}}\right\rangle, & \left\langle\psi_{\left(\vec{r}_{c}, t\right)}^{n, \vec{q}} \mid \psi_{\left(\vec{r}_{c}, t\right)}^{n^{\prime}, \vec{q}^{\prime}}\right\rangle=\delta_{n, n^{\prime}} \delta\left(\vec{q}-\vec{q}^{\prime}\right), \\
\left\langle\vec{r} \mid \psi_{\left(\vec{r}_{c}, t\right)}^{n, \vec{q}}\right\rangle=e^{i \vec{q} \cdot \vec{r}^{n}} u_{\left(\vec{r}_{c}, t\right)}^{n, \vec{r})}(\vec{r}), & u_{\left(\vec{r}_{c}, t\right)}^{n, \vec{r}}(\vec{r}+\vec{a})=u_{\left(\vec{r}_{c}, t\right)}^{n, \vec{r}}(\vec{r}),
\end{array}
$$

where the energy eigenvalues $E_{\left(\vec{r}_{c}, t\right)}^{n, \vec{q}}$ are labeled by the band index, $n$, and by the quasimomentum $\vec{q}$, restricted to the first Brillouin zone (IBZ). We assume that the time evolution of $\vec{r}_{c}$ closely follows the one obtained by the exact integration of the Schrödinger equation and that the eigenvalues $E_{\left(\vec{r}_{c}, t\right)}^{n, \vec{q}}$ form well separated bands, and that band jumping is forbidden. The label $n$ will be dropped in what follows.

A classical result by Karplus and Luttinger [66] says that

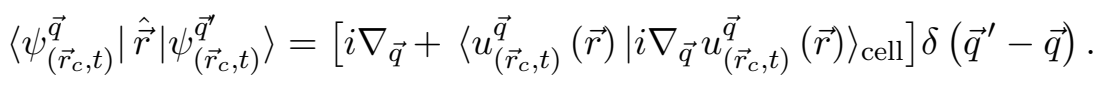

That is, the momentum representation of $\hat{\vec{r}}$ is

$$
\hat{\vec{r}}=i \nabla_{\vec{q}}+\overrightarrow{\mathcal{A}}\left(\vec{r}_{c}, \vec{q}, t\right), \quad \overrightarrow{\mathcal{A}}=\left\langle u_{\left(\vec{r}_{c}, t\right)}^{\vec{q}} \mid i \nabla_{\vec{q}} u_{\left(\vec{r}_{c}, t\right)}^{\vec{q}}\right\rangle_{\text {cell }},
$$

where $\langle\cdot \mid \cdot\rangle_{\text {cell }}$ is the restriction of the scalar product to the unit cell with periodic boundary conditions, and with normalization factor $(2 \pi)^{3} / V_{\text {cell }}$. Then the quantity $\overrightarrow{\mathcal{A}}\left(\vec{r}_{c}, \vec{q}, t\right)$ is interpreted as a $U(1)$ Berry connection, whose curvature appears in the commutation relations for the position operator components,

$$
\left[\hat{r}_{j}, \hat{r}_{l}\right]=i \epsilon_{j l} \partial_{q_{j}} \mathcal{A}_{l}\left(\vec{r}_{c}, \vec{q}, t\right)=\Theta_{j l}\left(\vec{r}_{c}, \vec{q}, t\right),
$$

which converts the dynamics of an ordinary particle in a periodic background potential into a quantum mechanical system in a non-commutative configuration space [14]. The antisymmetric tensor $\boldsymbol{\Theta}=\left(\Theta_{i j}\right)$ generalizes in fact the scalar parameter $\theta$ of the planar non-commutative theory.

Its effects cannot be disregarded for the semiclassical motion of a wave-packet

$$
\left|\widetilde{\Psi}\left[\vec{r}_{c}(t), \vec{q}_{c}(t)\right]\right\rangle=\int_{\mathrm{IBZ}} \Phi(\vec{q}, t)\left|\psi_{\left(\vec{r}_{c}, t\right)}^{\vec{q}}\right\rangle d \vec{q},
$$

built by superimposing one-band Bloch waves with a normalized amplitude $\Phi(\vec{q}, t)$. In fact, under the assumptions of small momentum dispersion, $\Delta_{q} \ll 2 \pi / l_{\text {latt }}$, it can be proved that the mean packet-position is

$$
\vec{r}_{c}(t)=\langle\widetilde{\Psi}|\hat{\vec{r}}| \widetilde{\Psi}\rangle \approx-\nabla_{\vec{q}_{c}} \arg \left[\Phi\left(\vec{q}_{c}, t\right)\right]+\overrightarrow{\mathcal{A}}\left(\vec{r}_{c}, \vec{q}_{c}, t\right),
$$

where the mean quasi-momentum,

$$
\vec{q}_{c}(t)=\int_{I B Z} \vec{q}|\Phi(\vec{q}, t)|^{2} d \vec{q},
$$


has been introduced. Then, the semiclassical description of the wave-packet is reduced to that of a particle - like system in the $\left(\vec{r}_{c}, \vec{q}_{c}\right)$ "phase space", the dynamics of which is obtained by minimizing the Schrödinger field action

$$
S=\int_{t_{1}}^{t_{2}}\left\{\frac{i}{2} \frac{\left\langle\Psi \mid \frac{d \Psi}{d t}\right\rangle-\left\langle\frac{d \Psi}{d t} \mid \Psi\right\rangle}{\langle\Psi \mid \Psi\rangle}-\frac{\langle\Psi|\hat{H}| \Psi\rangle}{\langle\Psi \mid \Psi\rangle}\right\} d t
$$

where $\left(\vec{r}_{c}(t), \vec{q}_{c}(t)\right)$ parametrize the wave-function [67]. This leads to an "approximate Lagrangian" for a point-like classical particle of the form (4.1), namely to

$$
\begin{aligned}
L_{\text {app }}= & \dot{\vec{r}}_{c} \cdot\left(\vec{q}_{c}+\overrightarrow{\mathcal{R}}\left(\vec{r}_{c}, \vec{q}_{c}, t\right)\right)+\dot{\vec{q}}_{c} \cdot \overrightarrow{\mathcal{A}}\left(\vec{r}_{c}, \vec{q}_{c}, t\right)+\mathcal{T}\left(\vec{r}_{c}, \vec{q}_{c}, t\right) \\
& -\mathcal{E}\left(\vec{r}_{c}, \vec{q}_{c}, t\right)-\Delta \mathcal{E}\left(\vec{r}_{c}, \vec{q}_{c}, t\right),
\end{aligned}
$$

where

$$
\begin{aligned}
& \mathcal{T}\left(\vec{r}_{c}, \vec{q}_{c}, t\right)=\left\langle u_{\left(\vec{r}_{c}, t\right)}^{\vec{q}_{c}} \mid i \partial_{t} u_{\left(\vec{r}_{c}, t\right)}^{\vec{q}_{c}}\right\rangle_{\text {cell }}, \\
& \overrightarrow{\mathcal{R}}\left(\vec{r}_{c}, \vec{q}_{c}, t\right)=\left\langle u_{\left(\vec{r}_{c}, t\right)}^{\vec{q}_{c}} \mid i \nabla_{\vec{r}_{c}} u_{\left(\vec{r}_{c}, t\right)}^{\vec{q}_{c}}\right\rangle_{\text {cell }}, \\
& \mathcal{E}=\left\langle\widetilde{\Psi}\left|\hat{H}_{\left(\vec{r}_{c}, t\right)}\right| \widetilde{\Psi}\right\rangle, \quad \Delta \mathcal{E}=\left\langle\widetilde{\Psi}\left|\hat{W}_{\left(\vec{r}_{c}, t\right)}\right| \widetilde{\Psi}\right\rangle .
\end{aligned}
$$

Together with $\overrightarrow{\mathcal{A}}$, the scalar $\mathcal{T}$ and the vector field $\overrightarrow{\mathcal{R}}$ provide us with the complete Berry connection on the entire "environmental parameter space" $\left(\vec{r}_{c}, \vec{q}_{c}, t\right)$. The quantity $\mathcal{E}$ expresses the potential energy felt by the wave packet in the periodic environment and $\Delta \mathcal{E}$ comes from the adiabatic perturbations.

For slowly changing electromagnetic potentials $\left(\vec{A}(\vec{r}, t), V_{e l}(\vec{r}, t)\right)$, the rather involved expressions above take an elegant form [22], - but the Bloch eigenfunctions get a gauge-dependent phase modification $\approx e \vec{A}\left(\vec{r}_{c}, t\right) \cdot \vec{r}$. In fact, a change of phase has no influence on the Berry connection because of (11.1), so one can introduce the gauge invariant kinetic momentum

$$
\vec{k}_{c}=\vec{q}_{c}-e \vec{A}\left(\vec{r}_{c}, t\right)
$$

and set again

$$
\begin{aligned}
& \overrightarrow{\mathcal{A}}\left(\vec{r}_{c}, \vec{q}_{c}, t\right)=\overrightarrow{\mathcal{A}}\left(\vec{k}_{c}\right), \\
& \overrightarrow{\mathcal{R}} \simeq-\left.e \nabla_{\vec{r}_{c}}\left(\vec{A}\left(\vec{r}_{c}, t\right) \cdot \vec{r}\right)\right|_{\vec{r}=\vec{r}_{c}}, \quad \mathcal{T} \simeq-e \partial_{t} \vec{A}\left(\vec{r}_{c}, t\right) \cdot \vec{r}_{c}, \\
& \mathcal{E}=\mathcal{E}_{0}\left(\vec{k}_{c}\right)+e V_{e l}\left(\vec{r}_{c}, t\right), \quad \Delta \mathcal{E}=-\vec{M}\left(\vec{k}_{c}, t\right) \cdot \vec{B}\left(\vec{r}_{c}, t\right),
\end{aligned}
$$

where

$$
\vec{M}\left(\vec{k}_{c}, t\right)=-\frac{e}{2 m}\langle\widetilde{\Psi}|\hat{\vec{L}}| \widetilde{\Psi}\rangle
$$

is the mean magnetic moment of the wave-packet. $\vec{B}\left(\vec{r}_{c}, t\right)$ and $\vec{E}\left(\vec{r}_{c}, t\right)$ are defined as usual from the mean values of the potentials. Dropping the label $c$, and putting

$$
\Theta_{i}=\frac{1}{2} \epsilon_{i j k} \Theta_{j k},
$$

the generalized semiclassical equations of motion are

$$
\begin{aligned}
& \dot{\vec{r}}=\nabla_{\vec{k}}\left[\mathcal{E}_{0}(\vec{k})-\vec{M}(\vec{k}, t) \cdot \vec{B}(\vec{r}, t)\right]-\dot{\vec{k}} \times \vec{\Theta}(\vec{k}), \\
& \dot{\vec{k}}=-e(\dot{\vec{r}} \times \vec{B}(\vec{r}, t)+\vec{E}(\vec{r}, t))+\nabla_{\vec{r}}(\vec{M}(\vec{k}, t) \cdot \vec{B}(\vec{r}, t)),
\end{aligned}
$$


further confirming the idea of the non-commutativity parameter, now a function of momentumspace variables, is in fact a Berry phase effect.

Notice, here that the semiclassical procedure has consistently "averaged" on the gauge degrees of freedom at local scales of order $\sim l_{w p}$, but the final model still possesses the same gauge invariant character as a point-like particle interacting with an external e.m. field.

Then, for the electronic wave-packet semiclassically described by (11.5), one can adapt the symplectic techniques described in the previous sections and it can be used for a Hamiltonian formulation.

\subsection{Hamiltonian structure}

Comparing the system (11.5) with the previous ones in (2.3) or (8.6), one recognizes a general common structure. The nice group-geometrical symmetry properties of the 2D Galilei group, which partially motivated the present research, are broken in general. However, the unifying framework for such differential systems is provided by the same ideology adopted in Sections 2 and 6, i.e. writing them as the kernel of a postulated anti-symmetric, closed, constant-rank Lagrange-Souriau 2-form $\sigma$ of the form

$$
\begin{aligned}
\sigma= & {\left[\left(1-Q_{i}\right) d q_{i}-e E_{i} d t\right] \wedge\left(d r_{i}-g_{i} d t\right) } \\
& +\frac{1}{2} e \epsilon_{i j k} B_{k} d r_{i} \wedge d r_{j}+\frac{1}{2} \epsilon_{i j k} \Theta_{k} d q_{i} \wedge d q_{j}+Q_{0} \epsilon_{i j} d r_{i} \wedge d q_{j},
\end{aligned}
$$

where the Souriau's prescription to explicitly include the electromagnetic contributions has been used. The vector fields $\vec{g}, \vec{\Theta}, \vec{Q}$ and the scalar functions $Q_{0}$ may depend on all independent variables $(\vec{r}, \vec{q}, t)$. Notice that (11.6) only contains "forces", i.e. gauge invariant quantities. Moreover, the so called "Maxwell principle" [3], i.e. the closure relation $d \sigma=0$, implies a set of integrability conditions for functions involved, which reduce to the usual Maxwell equations for $(\vec{E}, \vec{B})$, when the new extra fields are set to constants. Even in this case, and in 2 space dimensions, the resulting equations are non-trivial, coinciding for instance with (2.3) after the identifications $r_{i} \rightarrow x_{i}, g_{i} \rightarrow q_{i} / m, \theta_{3} \rightarrow \theta$ and $Q_{i} \equiv 0$.

We note (like in Section 2) that a model defined by the 2-form (11.6) may not possess a globally defined configuration space Lagrangian. This makes the value of the semiclassical Lagrangian (11.4) questionable. If it is assumed valid at least locally, the physical meaning of the coefficients appearing in (11.6) can be deduced, via exterior derivative, from the Cartan 1 -form

$$
\lambda=(\vec{q}+\overrightarrow{\mathcal{R}}) \cdot d \vec{r}+\overrightarrow{\mathcal{A}} \cdot d \vec{q}+(\mathcal{T}-\mathcal{E}-\Delta \mathcal{E}) d t .
$$

Thus, the most general equations of motion deriving from (11.4) (or equivalently from (11.6)) are

$$
\begin{aligned}
& (1+\boldsymbol{\Xi}) \dot{\vec{r}}+\Theta \dot{\vec{q}}=\nabla_{\vec{q}}[\mathcal{E}+\Delta \mathcal{E}-\mathcal{T}]+\partial_{t} \overrightarrow{\mathcal{A}}, \\
& X \dot{\vec{r}}+(1+\boldsymbol{\Xi}) \dot{\vec{q}}=-\nabla_{\vec{r}}[\mathcal{E}+\Delta \mathcal{E}-\mathcal{T}]-\partial_{t} \overrightarrow{\mathcal{R}},
\end{aligned}
$$

where the antisymmetric matrices $\boldsymbol{\Xi}=\left(\Xi_{i j}\right)$ and $\mathbf{X}=\left(X_{i j}\right)$ have elements

$$
\boldsymbol{\Xi}_{i j}=\partial_{r_{i}} \mathcal{A}_{j}-\partial_{q_{j}} \mathcal{R}_{i}, \quad X_{i j}=\partial_{r_{i}} \mathcal{R}_{j}-\partial_{r_{j}} \mathcal{R}_{i}
$$

The dynamical system (11.7) is defined on the tangent manifold of the configuration space, endowed with generalized coordinates $\vec{\xi}=(\vec{r}, \vec{q})$. But, when $\partial_{t} \overrightarrow{\mathcal{A}}=\partial_{t} \overrightarrow{\mathcal{R}} \equiv 0$, the rearrangement $\sigma=\omega-d H \wedge d t$ of the terms in (11.6) is possible, introducing the symplectic 2-form

$$
\omega=\left(\delta_{i, j}+\Xi_{i j}\right) d r_{i} \wedge d q_{j}+\frac{1}{2}\left[X_{i j} d q_{i} \wedge d q_{j}-\Theta_{i j} d r_{i} \wedge d r_{j}\right]
$$

and the Hamiltonian function $\mathcal{H}=\mathcal{E}+\Delta \mathcal{E}-\mathcal{T}$. 
Actually, the closure of $\sigma$ implies that, $d \omega=0$, for $\omega$. Equivalently, the set of differential constraints

$$
\begin{array}{ll}
\varepsilon_{i j k} \partial_{q_{i}} \Theta_{j k}=0, & \varepsilon_{i j k} \partial_{r_{i}} X_{j k}=0, \\
\partial_{q_{j}} \Xi_{i j}=-\partial_{r_{j}} \Theta_{i j}, & \partial_{r_{j}} \Xi_{i j}=\partial_{q_{j}} X_{i j}, \\
\left(1-\delta_{h k}\right) \varepsilon_{k i j} \partial_{q_{k}} \Xi_{i j}=\varepsilon_{h i j} \partial_{r_{h}} \Theta_{i j}, & \left(1-\delta_{h k}\right) \varepsilon_{k i j} \partial_{r_{k}} \Xi_{i j}=-\varepsilon_{h i j} \partial_{q_{h}} X_{i j},
\end{array}
$$

which, however, are automatically satisfied, because of the antisymmetry and the differentiability properties of the tensors $\boldsymbol{\Theta}, \boldsymbol{\Xi}$ and $\mathbf{X}$ defined in (11.2) and (11.8). Thus, for non degenerate $\omega=\omega_{\alpha \beta} d \xi_{\alpha} \wedge d \xi_{\beta}$, Poisson brackets,

$$
\{f, g\}=\omega^{\alpha \beta} \partial_{\alpha} f \partial_{\beta} g
$$

can be defined for any pair of functions $f(\vec{\xi})$ and $g(\vec{\xi})$, where $\omega^{\alpha \gamma} \omega_{\gamma \beta}=\delta_{\beta}^{\alpha}$ is the inverse of the symplectic matrix [3, 68]. Thus, the equations (11.7) take the usual Hamiltonian form $\dot{\xi}_{\alpha}=\left\{\xi_{\alpha}, \mathcal{H}\right\}$. In the present case $\left(\omega_{\alpha \beta}\right)$ is a real symplectic $6 \times 6$ matrix, which is non degenerate when

$$
\sqrt{\operatorname{det}\left(\omega_{\alpha \beta}\right)}=1-\frac{1}{2} \operatorname{Tr}\left(\boldsymbol{\Xi}^{2}+\mathbf{X}(\mathbf{1}+2 \boldsymbol{\Xi}) \boldsymbol{\Theta}\right) \neq 0
$$

Such a factor generalizes the denominators present in the Poisson brackets (2.5), (4.3) or (8.4). Moreover, it crucially appears in the expression of the invariant phase-space volume, ensuring the validity of the Liouville theorem [69, 64].

As special example, we deal with only momentum (gauge invariant) dependent Berry curvature $\vec{\Theta}(\vec{q})$ which is to be divergence-free according to the first equation in (11.9). That condition can be satisfied, except in one point, e.g., by a monopole in $\vec{q}$-space,

$$
\vec{\Theta}=g \frac{\vec{q}}{q^{3}},
$$

which is indeed the only possibility consistent with the spherical symmetry and the canonical relations $\left\{x_{i}, q_{j}\right\}=\delta_{i j}[65]$. The expression (11.10) appears to be consistent, at least qualitatively, with the data reported in [23] and in Spin Hall Effects [24].

In absence of a magnetic field and taking, for simplicity, the energy band $\epsilon_{n}(\vec{q})$ to be parabolic, the equations (11.5) for become

$$
\dot{\vec{r}}=\vec{q}+\frac{e g}{q^{3}} \vec{E} \times \vec{q}, \quad \dot{\vec{q}}=-e \vec{E} .
$$

The anomalous term shifts the velocity and deviates, hence, the particle's trajectory perpendicularly to the electric field, just like in the anomalous Hall effect, see [23].

A similar pattern arises in optics $[25,26,27,28]$ : to first order in the gradient of the refractive index $n$, spinning light is approximately described by the equations

$$
\dot{\vec{r}} \approx \vec{p}-\frac{s}{\omega} \operatorname{grad}\left(\frac{1}{n}\right) \times \vec{p}, \quad \dot{\vec{p}} \approx-n^{3} \omega^{2} \operatorname{grad}\left(\frac{1}{n}\right),
$$

where $s$ denotes the photon's spin. In the first relation we recognize, once again, an anomalous velocity relation of the type (11.5). The new term makes the light's trajectory deviate from that predicted in ordinary geometrical optics, giving rise to the "optical Magnus effect" [25]. A manifestation of this is the displacement of the light ray perpendicularly to the plane of incidence at the interface of two media with different refraction index: this is the "Optical Hall Effect" [26, 27, 28]. 
Another nice illustration is provided by the non-commutative Kepler problem [13]. Choosing the non-commutative vector $\vec{\Theta}$ in the vertical direction,

$$
\Theta_{i}=\theta \delta_{i z}
$$

the 3D problem reduces to the "exotic" model presented in Section 2. Then the authors of [13] show that, for the Kepler potential $V \propto r^{-1}$ the perturbation due to non-commutativity induces the precession of the perihelion point of planetary orbit.

As yet another example, we would like to mention the recent work [70], in which it is shown that a particle with "monopole-type" noncommutativity (11.10) admits a conserved Runge-Lenz vector, namely

$$
\vec{K}=\vec{r} \times \vec{J}-\alpha \frac{\vec{q}}{q},
$$

provided the Hamiltonian is

$$
H=\frac{\vec{r}^{2}}{2}+\frac{g^{2}}{2 q^{2}}+\frac{\alpha}{q}
$$

Note that $\vec{q}$ here is the momentum: the "monopole" is in "dual space". Let us observe that this expression is reminiscent of the Chern-Simons mechanics" [32] in that it has no mass term. The associated the equations of motion read,

$$
\dot{\vec{r}}=-\left(\frac{g^{2}}{q^{4}}+\frac{\alpha}{q^{3}}\right) \vec{q}+g \frac{\vec{q} \times \vec{r}}{q^{3}}, \quad \dot{\vec{q}}=-\vec{r} .
$$

The Kepler-type dynamical symmetry then allows one to show that the classical motions follow (arcs of) oblique ellipses [70].

\section{Acknowledgments}

This review also includes results obtained jointly with C. Duval, Z. Horváth, J. Lukierski, M. Plyushchay and W.J. Zakrzewski, to whom we express our indebtedness. L.M. thanks the INFN - Sezione of Lecce for partial financial support under the project LE41.

\section{References}

[1] Landau L., Lifschitz E., Physique théorique, Tome III, Mécanique quantique. Théorie non relativiste, Mir, Moscow, 1967.

[2] Simms D., Projective representations, symplectic manifolds and extensions of Lie algebras, Lectures given at Centre de Physique Théorique, CNRS, Marseille (1969), Preprint 69/P.300.

Aldaya V., de Azcárraga J.A., Cohomology, central extensions, and (dynamical) groups, Internat. J. Theoret. Phys. 24 (1985), 141-154.

Tuynman G.M., Wiegerinck W.A.J.J., Central extensions and physics, J. Geom. Phys. 4 (1987), $207-258$.

Marmo G., Morandi G., Simoni A., Sudarshan E.C.G., Quasi-invariance and central extensions, Phys. Rev. D 37 (1988), 2196-2205.

de Saxcé G., Vallée C., Construction of a central extension of a Lie group from its class of symplectic cohomology, J. Geom. Phys. 60 (2010), 165-174.

[3] Souriau J.-M., Structure des systèmes dynamiques, Dunod, Paris, 1970.

Souriau J.-M., Structure of dynamical systems. A symplectic view of physics, Progress in Mathematics, Vol. 149, Birkhäuser Boston, Inc., Boston, MA, 1997.

[4] Bargmann V., On unitary ray representations of continuous groups, Ann. of Math. (2) 59 (1954), 1-46.

[5] Lévy-Leblond J.-M., Galilei group and Galilean invariance, in Group Theory and Applications, Vol. II, Academic Press, New York, 1971, 221-299. 
[6] Duval C., Exotic Galilei group, IQHE and Chern-Simons electrodynamics, Unpublished draft, 1995. Grigore D.R., Transitive symplectic manifolds in 1+2 dimensions, J. Math. Phys. 37 (1996), 240-253. Grigore D.R., The projective unitary irreducible representations of the Galilei group in $1+2$ dimensions, J. Math. Phys. 37 (1996), 460-473, hep-th/9312048.

[7] Ballesteros A., Gadella M., del Olmo M.A., Moyal quantization of $2+1$-dimensional Galilean systems, J. Math. Phys. 33 (1992), 3379-3386.

Brihaye Y., Gonera C., Giller S., Kosiński P., Galilean invariance in $2+1$ dimensions, hep-th/9503046.

[8] Lukierski J., Stichel P.C., Zakrzewski W.J., Galilean-invariant $(2+1)$-dimensional models with a ChernSimons-like term and $D=2$ noncommutative geometry, Ann. Physics 260 (1997), 224-249, hep-th/9612017.

[9] Duval C., Horváthy P.A., The exotic Galilei group and the "Peierls substitution", Phys. Lett. B 479 (2000), 284-290, hep-th/0002233.

Duval C., Horváthy P.A., Exotic Galilean symmetry in the non-commutative plane and the Hall effect, J. Phys. A: Math. Gen. 34 (2001), 10097-10107, hep-th/0106089.

Horváthy P.A., The non-commutative Landau problem, Ann. Physics 299 (2002), 128-140, hep-th/0201007.

[10] Nair V.P., Polychronakos A.P., Quantum mechanics on the noncommutative plane and sphere, Phys. Lett. B 505 (2001), 267-274, hep-th/0011172.

[11] Sochichiu C., A note on noncommutative and false noncommutative spaces, Appl. Sciences 3 (2001), 48-51, hep-th/0010149.

Bellucci S., Nersessian A., Sochichiu C., Two phases of the non-commutative quantum mechanics, Phys. Lett. B 522 (2001), 345-349, hep-th/0106138.

Acatrinei C., Path integral formulation of noncommutative quantum mechanics, J. High Energy Phys. 2001 (2001), no. 9, 007, 7 pages, hep-th/0107078.

Gamboa J., Loewe M., Méndez F., Rojas J.C., Noncommutative quantum mechanics, Phys. Rev. D 64 (2001), 067901, 3 pages.

Banerjee R., A novel approach to noncommutativity in planar quantum mechanics, Modern Phys. Lett. A 17 (2002), 631-645, hep-th/0106280.

[12] Djemai A.E.F., Smail H., On quantum mechanics on noncommutative quantum phase space, Commun. Theor. Phys. 41 (2004), 837-844, hep-th/0309006.

Banerjee R., Kumar K., Deformed relativistic and nonrelativistic symmetries on canonical noncommutative spaces, Phys. Rev. D 75 (2007), 045008, 5 pages, hep-th/0604162.

Banerjee R., Deformed Schrödinger symmetry on noncommutative space, Eur. Phys. J. C 47 (2006), 541545, hep-th/0508224.

Papageorgiou G., Schroers B.J., A Chern-Simons approach to Galilean quantum gravity in $2+1$ dimensions, J. High Energy Phys. 2009 (2009), no. 11, 009, 40 pages, arXiv:0907.2880.

Scholtz F.G., Gouba L., Hafver A., Rohwer C.M., Formulation, interpretation and application of noncommutative quantum mechanics, J. Phys. A: Math. Theor. 42 (2009), 175303, 13 pages, arXiv:0812.2803. Gangopadhyay S., Scholtz F.G., Path integral action of a particle in the non commutative plane, arXiv:0904.0379.

Baldiotti M.C., Gazeau J.P., Gitman D.M., Semiclassical and quantum description of motion on noncommutative plane, Phys. Lett. A 373 (2009), 3937-3943, arXiv:0906.0388.

Gomes M., Kupriyanov V.G., Position-dependent noncommutativity in quantum mechanics, Phys. Rev. D 79 (2009), 125011, 6 pages, arXiv:0902.3252.

[13] Romero J.M., Santiago J.A., Vergara J.D., Newton's second law in a non-commutative space, Phys. Lett. A 310 (2003), 9-12, hep-th/0211165.

Romero J.M., Vergara J.D., The Kepler problem and noncommutativity, Modern Phys. Lett. A 18 (2003), 1673-1680, hep-th/0303064.

[14] Szabo R.J., Quantum field theory on noncommutative spaces, Phys. Rep. 378 (2003), 207-299, hep-th/0109162.

[15] Delduc F., Duret Q., Gieres F., Lefrancois M., Magnetic fields in noncommutative quantum mechanics, J. Phys. Conf. Ser. 103 (2008), 012020, 26 pages, arXiv:0710.2239.

[16] Jackiw R., Nair V.P., Anyon spin and the exotic central extension of the planar Galilei group, Phys. Lett. B 480 (2000), 237-238, hep-th/0003130.

Duval C., Horváthy P.A., Spin and exotic Galilean symmetry, Phys. Lett. B 547 (2002), 306-312, hep-th/0209166.

[17] Fehér L., Equations of motion and dynamical symmetries of point particles, PhD thesis, University of Szeged, 1988 (in Hungarian).

Skagerstam B.-S., Stern A., Topological quantum mechanics in $2+1$ dimensions, Internat. J. Modern Phys. A 5 (1990), 1575-1595. 
[18] Plyushchay M.S., Relativistic model of the anyon, Phys. Lett. B 248 (1990), 107-112.

Plyushchay M.S., Fractional spin. Majorana-Dirac field, Phys. Lett. B 273 (1991), 250-254.

Jackiw R., Nair V.P., Relativistic wave equation for anyons, Phys. Rev. D 43 (1991), 1933-1942.

[19] Horváthy P.A., Plyushchay M.S., Non-relativistic anyons, exotic Galilean symmetry and noncommutative plane, J. High Energy Phys. 2002 (2002), no. 6, 033, 11 pages, hep-th/0201228.

Horváthy P.A., Mathisson's spinning electron: noncommutative mechanics and exotic Galilean symmetry, 66 years ago, Acta Phys. Polon. B 34 (2003), 2611-2621, hep-th/0303099.

Plyushchay M.S., Majorana equation and exotics: higher derivative models, anyons and noncommutative geometry, Electron. J. Theor. Phys. 3 (2006), no. 10, 17-31, math-ph/0604022.

[20] Horváthy P.A., Plyushchay M.S., Anyon wave equations and the noncommutative plane, Phys. Lett. B 595 (2004), 547-555, hep-th/0404137.

Horváthy P.A., Plyushchay M.S., Valenzuela M., Bosons, fermions and anyons in the plane, and supersymmetry arXiv:1001.0274.

[21] Horváthy P.A., Martina L., Stichel P.C., Galilean symmetry in noncommutative field theory, Phys. Lett. B 564 (2003), 149-154, hep-th/0304215.

Horváthy P.A., Martina L., Stichel P.C., Galilean noncommutative gauge theory: symmetries and vortices, Nuclear Phys. B 673 (2003), 301-318, hep-th/0306228.

[22] Chang M.C., Niu Q., Berry phase, hyperorbits, and the Hofstadter spectrum, Phys. Rev. Lett. 75 (1995), 1348-1351, cond-mat/9505021.

Bohm A., Mostafazadeh A., Koizumi H., Niu Q., Zwanziger J., The geometric phase in quantum systems. Foundations, mathematical concepts, and applications in molecular and condensed matter physics, Texts and Monographs in Physics, Springer-Verlag, Berlin, 2003, Chapter 12.

Xiao D., Chang M.C., Niu Q., Berry phase effects on electronic properties, Rev. Modern Phys. 82 (2010), 1959-2007, arXiv:0907.2021.

[23] Jungwirth T., Niu Q., MacDonald A.H., Anomalous Hall effect in ferromagnetic semiconductors, Phys. Rev. Lett. 88 (2002), 207208, 4 pages, cond-mat/0110484.

Culcer D., MacDonald A.H., Niu Q., Anomalous Hall effect in paramagnetic two-dimensional systems, Phys. Rev. B 68 (2003), 045327, 9 pages, cond-mat/0311147.

Fang Z. et al., The anomalous Hall effect and magnetic monopoles in momentum space, Science 302 (2003), no. 5642, 92-95, cond-mat/0310232.

Horváthy P.A., Anomalous Hall effect in noncommutative mechanics, Phys. Lett. A 359 (2006), 705-706, cond-mat/0606472.

[24] Murakami S., Nagaosa N., Zhang S.-C., Dissipationless quantum spin current at room temperature, Science 301 (2003), no. 5638, 1348-1351, cond-mat/0308167.

Sinova J., Culcer D., Niu Q., Sinitsyn N.A., Jungwirth T., MacDonald A.H., Universal intrinsic spin-Hall effect, Phys. Rev. Lett. 92 (2004), 126603, 4 pages, cond-mat/0307663.

Murakami S., Intrinsic spin Hall effect, Adv. Solid State Phys. 45 (2005), 197-209, cond-mat/0504353.

[25] Liberman V.S., Zeldovich B.Ya., Spin-orbit interaction of a photon in an inhomogeneous medium, Phys. Rev. A 46 (1992), 5199-5207.

Bliokh K.Yu., Bliokh Yu.P., Optical Magnus effect as a consequence of Berry phase anisotropy, JETP Lett. 79 (2004), 519-522.

Bliokh K.Yu., Bliokh Yu.P., Topological spin transport of photons: the optical Magnus effect and Berry phase, Phys. Lett. A 333 (2004), 181-186, physics/0402110.

Sadykov N.R., Twistability of spin particle trajectories, Theoret. and Math. Phys. 135 (2003), 685-692.

Bérard A., Mohrbach H., Spin Hall effect and Berry phase of spinning particles, Phys. Lett. A 352 (2006), 190-195, hep-th/0404165.

[26] Onoda M., Murakami S., Nagaosa N., Hall effect of light, Phys. Rev. Lett. 93 (2004), 083901, 4 pages, cond-mat/0405129.

Bliokh K.Y., Niv A., Kleiner V., Hasman E., Geometrodynamics of spinning light, Nature Photon. 2 (2008), 748-753, arXiv:0810.2136.

[27] Duval C., Horváth Z., Horváthy P.A., Geometrical spinoptics and the optical Hall effect, J. Geom. Phys. 57 (2007), 925-941, math-ph/0509031.

Duval C., Horváth Z., Horváthy P.A., Fermat principle for spinning light. Phys. Rev. D 74 (2006), 021701, 5 pages, cond-mat/0509636.

[28] Bliokh K.Y., Geometrodynamics of polarized light: Berry phase and spin Hall effect in a gradient-index medium, J. Opt. A: Pure Appl. Opt. 11 (2009), 094009, 14 pages, arXiv:0903.1910.

[29] Horváthy P.A., Exotic galilean symmetry and non-commutative mechanics in mathematical and in condensed matter physics, hep-th/0602133. 
[30] Martina L., Hamiltonian theory of anyons in crystals, J. Math. Sci. 151 (2008), 3159-3166.

[31] Stichel P.C., Dynamics and symmetries on the noncommutative plane, hep-th/0611172.

[32] Dunne G.V., Jackiw R., Trugenberger C.A., "Topological" (Chern-Simons) quantum mechanics, Phys. Rev. D 41 (1990), 661-666.

Dunne G., Jackiw R., "Peierls substitution" and Chern-Simons quantum mechanics, Nuclear Phys. B Proc. Suppl. 33C (1993), 114-118.

[33] Laughlin R.B., Anomalous quantum Hall effect: an incompressible quantum fluid with fractionally charged excitations, Phys. Rev. Lett. 50 (1983), 1395-1398.

Stone M. (Editor), Quantum Hall effect, World Scientific Publishing Co., Inc., River Edge, NJ, 1992.

[34] Chaichian M., Ghosh S., Langvik M., Tureanu A., Dirac quantization condition for monopole in noncommutative space-time, Phys. Rev. D 79 (2009), 125029, 5 pages, arXiv:0902.2453.

[35] Mendes A.C.R., Neves C., Oliveira W., Takakura F.I., A new approach to canonical quantization of the radiation damping, Eur. Phys. J. C 45 (2006), 257-261, hep-th/0503135.

[36] Snyder H.S., Quantized space-time, Phys. Rev. 71 (1947), 38-41.

[37] Ghosh S., A novel "magnetic" field and its dual non-commutative phase space, Phys. Lett. B 638 (2006), 350-355, hep-th/0511302.

[38] Lukierski J., Ruegg H., Nowicki A., Tolstoy V.N., q-deformation of Poincaré algebra, Phys. Lett. B 264 (1991), 331-338.

[39] Amelino-Camelia G., Fundamental physics in space: a quantum-gravity perspective, Gen. Relativity Gravitation 36 (2004), 539-560, astro-ph/0309174.

[40] Giller S., Kosiński P., Majewski M., Maślanka P., Kunz J., More about the q-deformed Poincaré algebra, Phys. Lett. B 286 (1992), 57-62.

[41] de Azcárraga J.A., Pérez Bueno J.C., Relativistic and Newtonian א-space-times, J. Math. Phys. 36 (1995), 6879-6896, q-alg/9505004.

[42] Plyushchay M.S., Relativistic particle with torsion, Majorana equation and fractional spin, Phys. Lett. B 262 (1991), 71-78.

Plyushchay M.S., The model of relativistic particle with torsion, Nuclear Phys. B 362 (1991), 54-72.

[43] Alvarez P.D., Gomis J., Kamimura K., Plyushchay M.S., (2+1)D exotic Newton-Hooke symmetry, duality and projective phase, Ann. Physics 322 (2007), 1556-1586, hep-th/0702014.

Alvarez P.D., Gomis J., Kamimura K., Plyushchay M.S., Anisotropic harmonic oscillator, non-commutative Landau problem and exotic Newton-Hooke symmetry, Phys. Lett. B 659 (2008), 906-912, arXiv:0711.2644. del Olmo M.A., Plyushchay M.S., Electric Chern-Simons term, enlarged exotic Galilei symmetry and noncommutative plane, Ann. Physics 321 (2006), 2830-2848, hep-th/0508020.

[44] Negro J., del Olmo M.A., Tosiek J., Anyons, group theory and planar physics, J. Math. Phys. 47 (2006), 033508, 19 pages, math-ph/0512007.

[45] Chou C., Nair V.P., Polychronakos A.P, On the electromagnetic interaction of anyons, Phys. Lett. B 304 (1993), 105-110, hep-th/9301037.

[46] Ghosh S., Spinning particles in $2+1$ dimensions, Phys. Lett. B 338 (1994), 235-240, hep-th/9406089.

Ghosh S., Anyons in an electromagnetic field and the Bargmann-Michel-Telegdi equation, Phys. Rev. D 51 (1995), 5827-5829, hep-th/9409169.

Banerjee N., Ghosh S., Interacting anyons and the Darwin Lagrangian, Phys. Rev. D 52 (1995), 6130-6133, hep-th/9412022.

Basu B., Ghosh S., Dhar S., Noncommutative geometry and geometric phases, Europhys. Lett. 76 (2006), 395-401, hep-th/0604068.

Dhar S., Basu B., Ghosh S., Spin Hall effect for anyons, Phys. Lett. A 371 (2007), 406-409, cond-mat/0701096.

Cortés J.L., Gamboa J., Velázquez L., Electromagnetic interaction of anyons in non-relativistic quantum field theory, Internat. J. Modern Phys. A 9 (1994), 953-968, hep-th/9211106.

Cortés J.L., Plyushchay M.S., Anyons as spinning particles, Internat. J. Modern Phys. A 11 (1996), 33313362, hep-th/9505117.

[47] Maude D.K., Potemski M., Portal J.C., Henini M., Eaves L., Hill G., Pate M.A., Spin excitations of a twodimensional electron gas in the limit of vanishing Landé $g$ factor, Phys. Rev. Lett. 77 (1996), 4604-4607. Leadley D.R., Nicholas R.J., Maude D.K., Utjuzh A.N., Portal J.C., Harris J.J., Foxon C.T., Fractional quantum Hall effect measurements at zero $g$ factor, Phys. Rev. Lett. 79 (1997), 4246-4249, cond-mat/9706157. 
[48] Duval C., Horváthy P.A., Anyons with anomalous gyromagnetic ratio and the Hall effect, Phys. Lett. B 594 (2004), 402-409, hep-th/0402191.

Duval C., Horváthy P.A., Noncommuting coordinates, exotic particles, and anomalous anyons in the Hall effect, Theoret. and Math. Phys. 144 (2005), 899-906, hep-th/0407010.

[49] Duval C., Fliche H.-H., Souriau J.-M., Un modèle de particule à spin dans le champ gravitationnel et électromagnétique, C. R. Acad. Sci. Paris Sér. A-B 274 (1972), A1082-A1084.

Duval C., Un modèle de particule à spin dans le champ électromagnétique et gravitationnel extérieure, Thèse de $3^{e}$ cycle. Marseille, 1972.

[50] Souriau J.-M., Modèle de particule à spin dans le champ électromagnétique et gravitationnel, Ann. Inst. H. Poincaré Sect. A (N.S.) 20 (1974), 315-364.

Duval C., The general relativistic Dirac-Pauli particle: an underlying classical model, Ann. Inst. H. Poincaré Sect. A (N.S.) 25 (1976), 345-362.

[51] Horváthy P.A., Plyushchay M.S., Nonrelativistic anyons in external electromagnetic field, Nuclear Phys. B 714 (2005), 269-291, hep-th/0502040.

[52] Lukierski J., Stichel P.C., Zakrzewski W.J., Noncommutative planar particle dynamics with gauge interactions, Ann. Physics 306 (2003), 78-95, hep-th/0207149.

[53] Fosco C.D., Torroba G., Noncommutative theories and general coordinate transformations, Phys. Rev. D 71 (2005), 065012, 9 pages, hep-th/0409240.

[54] Acatrinei C.S., A simple signal of noncommutative space, Modern Phys. Lett. A 20 (2005), 1437-1441, hep-th/0311134.

[55] Lukierski J., Stichel P.C., Zakrzewski W.J., $N=2$ supersymmetric planar particles and magnetic interactions from noncommutativity, Phys. Lett. B 602 (2004), 249-254, hep-th/0407247.

[56] Alvarez P.D., Cortés J.L., Horváthy P.A., Plyushchay M.S., Super-extended noncommutative Landau problem and conformal symmetry, J. High Energy Phys. 2009 (2009), no. 3, 034, 14 pages, arXiv:0901.1021.

[57] Horváthy P.A., Plyushchay M.S., Valenzuela M., Bosonized supersymmetry of anyons and supersymmetric exotic particle on the non-commutative plane, Nuclear Phys. B 768 (2007), 247-262, hep-th/0610317.

[58] Zhang S.C., Hansson T.H., Kivelson S., Effective-field-theory model for the fractional quantum Hall effect, Phys. Rev. Lett. 62 (1989), 82-85.

Zhang S.C., The Chern-Simons-Landau-Ginzburg theory of the fractional quantum Hall effect, Internat. J. Modern Phys. B 6 (1992), 25-58.

[59] Lozano G.S., Moreno E.F., Schaposnik F.A., Self-dual Chern-Simons solitons in non-commutative space, J. High Energy Phys. 2001 (2001), no. 2, 036, 17 pages, hep-th/0012266.

Bak D., Kim S.K., Soh K.-S., Yee J.H., Noncommutative Chern-Simons solitons, Phys. Rev. D 64 (2001), 025018, 9 pages, hep-th/0102137.

[60] Jackiw R., Pi S.-Y., Soliton solutions to the gauged nonlinear Schrödinger equation on the plane, Phys. Rev. Lett. 64 (1990), 2969-2972.

Jackiw R., Pi S.-Y., Classical and quantal nonrelativistic Chern-Simons theory, Phys. Rev. D 42 (1990), $3500-3513$.

Jackiw R., Pi S.-Y., Self-dual Chern-Simons solitons, Prog. Theor. Phys. Suppl. (1992), no. 107, 1-40.

Dunne G., Self-dual Chern-Simons theories, Springer Lecture Notes in Physics, Vol. 36, Springer, New York, 1995.

Horváthy P.A., Zhang P., Vortices in (abelian) Chern-Simons gauge theory, Phys. Rep. 481 (2009), 83-142, arXiv:0811.2094.

[61] Hadasz L., Lindström U., Roçek M., von Unge R., Time dependent solitons of noncommutative ChernSimons theory coupled to scalar fields, Phys. Rev. D 69 (2004), 105020, 12 pages, hep-th/0309015.

Horváthy P.A., Stichel P.C., Moving vortices in noncommutative gauge theory, Phys. Lett. B 583 (2004), 353-356, hep-th/0311157.

[62] Ashcroft N.W., Mermin N.D., Solid state physics, Saunders, Philadelphia, 1976.

[63] Horváthy P.A., Martina L., Stichel P.C., Enlarged Galilean symmetry of anyons and the Hall effect, Phys. Lett. B 615 (2005), 87-92, hep-th/0412090.

[64] Duval C., Horváth Z., Horváthy P.A., Martina L., Stichel P.C., Berry phase correction to electron density in solids and exotic dynamics, Modern Phys. Lett. B 20 (2006), 373-378, cond-mat/0506051.

Duval C., Horváth Z., Horváthy P.A., Martina L., Stichel P.C., Comment on "Berry phase correction to electron density in solids" by Xiao et al., Phys Rev. Lett. 96 (2006), 099701, 1 page.

Stone M., Mathematical methods II, available at http://w3.physics.uiuc. edu/ $\sim \mathrm{m}-\mathrm{stone} 5 / \mathrm{mmb} / \mathrm{mmb} . \mathrm{html}$. 
Bliokh K.Yu., On Hamiltonian nature of semiclassical motion equations in the presence of electromagnetic field and Berry curvature, Phys. Lett. A 351 (2006), 123-124, cond-mat/0507499.

Gosselin P., Menas F., Bérard A., Mohrbach H., Semiclassical dynamics of electrons in magnetic Bloch bands: an Hamiltonian approach, Europhys. Lett. 76 (2006), 651-656, cond-mat/0601472.

[65] Bérard A., Mohrbach H., Monopole and Berry phase in momentum space in noncommutative quantum mechanics, Phys. Rev. D 69 (2004), 127701, 4 pages, hep-th/0310167.

[66] Karplus R., Luttinger J.M., Hall effect in ferromagnetics, Phys. Rev. 95 (1954), 1154-1160.

[67] Kramer P., Saraceno M., Geometry of the time-dependent variational principle in quantum mechanics, Lecture Notes in Physics, Vol. 140, Springer-Verlag, Berlin - New York, 1981.

[68] Abraham R., Mardsen J., Foundations of mechanics, Addison-Wesley, Reading, 1978.

Marmo G., Saletan E.J., Simoni A., Vitale B., Dynamical systems. A differential geometric approach to symmetry and reduction, John Wiley \& Sons, Ltd., Chichester, 1985.

[69] Xiao D., Shi J.R., Niu Q., Berry phase correction to electron density of states in solids, Phys. Rev. Lett. 95 (2005), 137204, 4 pages, cond-mat/0502340.

[70] Zhang P.M., Horváthy P.A., Ngome J.P., Non-commutative oscillator with Kepler-type dynamical symmetry, arXiv:1006.1861. 